\title{
Asignación de recursos del Sistema General de Regalías, un análisis desde la perspectiva de la Dinámica de Sistemas
}

Resource Allocation of Royalty General System, an Analysis from System Dynamics' Perspective 


\title{
Asignación de recursos del Sistema General de Regalías, un análisis desde la perspectiva de la Dinámica de Sistemas ${ }^{1}$
}

\section{Resource Allocation of Royalty General System, an Analysis from System Dynamics' Perspective}

\author{
Germán Andrés Méndez², Rafael Fajardo ${ }^{3}$, Silvia Catalina Peña ${ }^{4}$, \\ Miguel Fajardo Carantón ${ }^{5}$ \\ 'Universidad Distrital Francisco José de Caldas, Bogotá D.C., Colombia
}

Artículo recibido en septiembre de 2016; artículo aceptado en noviembre de 2016

Citación del artículo: Méndez, G., Fajardo, R., Peña, S., \& Fajardo, M. (2017). Asignación de Recursos del Sistema

General de Regalías, un Análisis desde la perspectiva de Dinámica de Sistemas.

I+D Revista de Investigaciones, 9(1), 93 - 106

\begin{abstract}
Resumen
Este artículo muestra los avances de una investigación, dirigida al análisis de la formulación de política pública (PP), especialmente la distribución de recursos del Sistema General de Regalías (SGR) usando Dinámica de Sistemas. Primero, se presenta la situación actual del SGR resaltando que no siempre los recursos se asignan con el fin de satisfacer las necesidades básicas o urgentes de una población. Para un análisis más profundo de cómo podría desarrollarse este proceso, se procede a construir un modelo con dinámica de sistemas teniendo en cuenta aspectos técnicos, y cómo los expertos sugieren que debe realizarse la PP; a estos se suman los actores, tanto sociales, como políticos y privados, además de su interés particular. Luego, se describen los diagramas, resultado de la investigación y el modelo base propuesto para validarlo. Finalmente, se muestran los resultados obtenidos y la comparación con la realidad que desencadena la distribución actual.
\end{abstract}

Palabra clave: Dinámica de sistemas, política pública, Sistema General de Regalías en Colombia (Tesauro Spines).

\footnotetext{
Abstract

This paper shows development and progress of a research in process in the Universidad Distrital Francisco José de Caldas by Expert Systems and Simulation Group (E.S.S.), aimed to analysis of Public Policy (PP) formulation, specifically the allocation of resources of the General System royalties using System Dynamics. Presents first the current situation 1. Artículo de investigación, enfoque cuantitativo, resultado de un proyecto de investigación en curso, desarrollado en el grupo de investigación Sistemas Complejos y Simulación (SES) de la Universidad Distrital Francisco José de Caldas de la ciudad de Bogotá (Colombia): Dirección: Carrera 8\#40-62. PBX: (1) 3239300

2. Ingeniero Industrial, Universidad Distrital Francisco José de Caldas, Doctorado en Ciencias Técnicas, Universidad Central de las Villas (Cuba). Docente director del grupo de investigación de Sistemas Expertos y Simulación (SES) Universidad Distrital Francisco José de Caldas de la ciudad de Bogotá (Colombia): Dirección: Carrera 8 n. ${ }^{\circ} 40$ - 62. PBX: (1) 3239300. Correo institucional: gmendez@udistrital.edu.co

3. Ingeniero Industrial, Universidad Distrital Francisco José de Caldas. Master of Business Administration, Universidad de San Pablo CEU (España). Universidad Distrital Francisco José de Caldas de la ciudad de Bogotá (Colombia): Dirección: Carrera 8 \#40-62. Correo institucional: rafa.fajardo@ gmail.com

4. Estudiante de Ingeniería Industrial, Universidad Distrital Francisco José de Caldas. Integrante del grupo de investigación SES Universidad Distrital Francisco José de Caldas de la ciudad de Bogotá (Colombia): Dirección: Carrera 8 n. 40 - 62. Correo: silviacpa0620@gmail.com

5. Estudiante de Ingeniería Industrial, Universidad Distrital Francisco José de Caldas. Integrante del grupo de investigación SES Universidad Distrital Francisco José de Caldas de la ciudad de Bogotá (Colombia): Dirección: Carrera 8 n. 40 - 62. Correo: miky116@gmail.com
} 
of the General System of Royalties (SGR) identify that not always the resources are allocated in the order to satisfy the basic or urgent needs of a population. To perform a deeper analysis of how this process could be done, we proceed to perform a model with system dynamics which seeks a better approach to reality considering technical aspects, and how experts suggest that public policy should be performed; actors, both social, political and private, as well their interest. Then we describe the diagrams, result of the investigation and the base model proposed for validation. Finally we present the results and the comparison with reality that triggers the current distribution.

Keywords: System dynamics, public policy, Royalties System in Colombia.

Gran parte de la teoría de formulación de PP asume que esta puede ser diseñada a partir del modelamiento de los entornos sociales como sistemas simples. Sin embargo, gran parte de las debilidades en el diseño y la implementación de PP es que esta ha sido construida con un conocimiento limitado del entorno, es decir sin una visión completa y compleja de las necesidades, intereses y realidades de los territorios donde se adelantan dichas políticas, es así que una de las cuestiones que mayor dificultad presenta para los decisores es saber si los efectos de una decisión se acercarán a lo originalmente previsto, o mejor aún, si las consecuencias deseables superaran a las indeseables (Segura, 2009). Un correcto diseño debe contemplar aspectos como intereses, necesidades, debilidades, contexto socioeconómico, cultural y ambiental, principalmente, la interacción entre personas, que le aporta un carácter complejo.

En Colombia, una de las fuentes de financiación más relevantes de inversiones en PP son los recursos provenientes del SGR, el cual fue adoptado mediante la Ley 1530 de 2011 y se implementa en el año 2012.Este documento presenta, de manera resumida, el trabajo investigativo de análisis sobre el SGR realizado desde la perspectiva de la complejidad y la incertidumbre, usando como referencia la metodología del grupo de investigación Sistemas Expertos y Simulación (Méndez, Álvarez \& López, 2015) incorporando elementos de la Dinámica de Sistemas, técnica importante en la elaboración de políticas y en particular, de las políticas públicas.

\section{Comportamiento del SGR entre los años 2012 y 2015}

Para la regulación de los recursos del SGR, en Colombia se han agrupado los treinta y dos departamentos y sus municipios en siete grandes regiones, según la clasificación dictada por el Departamento Nacional de Planeación; dichas regiones han sido nombradas así: Caribe, Centro oriente, Centro sur, Cormagdalena, del Llano, Eje Cafetero y Pacífico.

Desde la fecha de inicio de implementación del nuevo SGR -2012- a diciembre de 2015, en Colombia se han aprobado 9020 proyectos, en los que se ha invertido más de diecinueve billones de pesos que equivalen a poco más de 9500 millones de dólares. Cada región contó con sumas diferentes de acuerdo a los recursos naturales no renovables que se explotan en sus departamentos, pero la región que más devenga no necesariamente es la que cuenta con mayor número de proyectos asignados, tal y como se puede observar en la Figura 1.

Ahora bien, el SGR ha definido que las inversiones a través de proyectos se realicen en el marco de veintiún sectores de entre los cuales se destaca el sector transporte, que suma aproximadamente USD\$3025 millones en 2798 proyectos, evidenciando que es el rubro en que más se invierte, salvo en Bogotá y Amazonas, en los que puntea el sector "Ciencia y Tecnología" con $78 \%$ y $36 \%$ del total de los proyectos, respectivamente.

A continuación, se detalla el análisis dela región Caribe, en la cual se comparan los planes de desarrollo departamental con la cantidad de proyectos y el dinero invertido, encontrando que no existe conexión entre lo propuesto en tales planes y los resultados. Por ejemplo, en el plan de desarrollo del departamento del Atlántico se asegura:

El núcleo del plan es la búsqueda de la solución a los problemas sociales del territorio, especialmente el ataque a la situación de pobreza y la inequidad social; de allí la importancia que toman aspectos como la vivienda, la salud, la educación y la nutrición (Gobernación del Atlántico, 2012).

Pero los sectores mencionados en este fragmento (vivienda, salud y nutrición) no hacen parte de los sectores con mayor inversión; esto se aprecia especialmente en el sector "Salud y protección social", que no cuenta con proyectos aprobados en este intervalo de tiempo, 20122015.

Lo mismo sucede en el caso de La Guajira, que, de acuerdo con la información del DNP (2015), es el departamento con el mayor índice de necesidades básicas insatisfechas y de mayor desigualdad en Colombia y en donde, según 


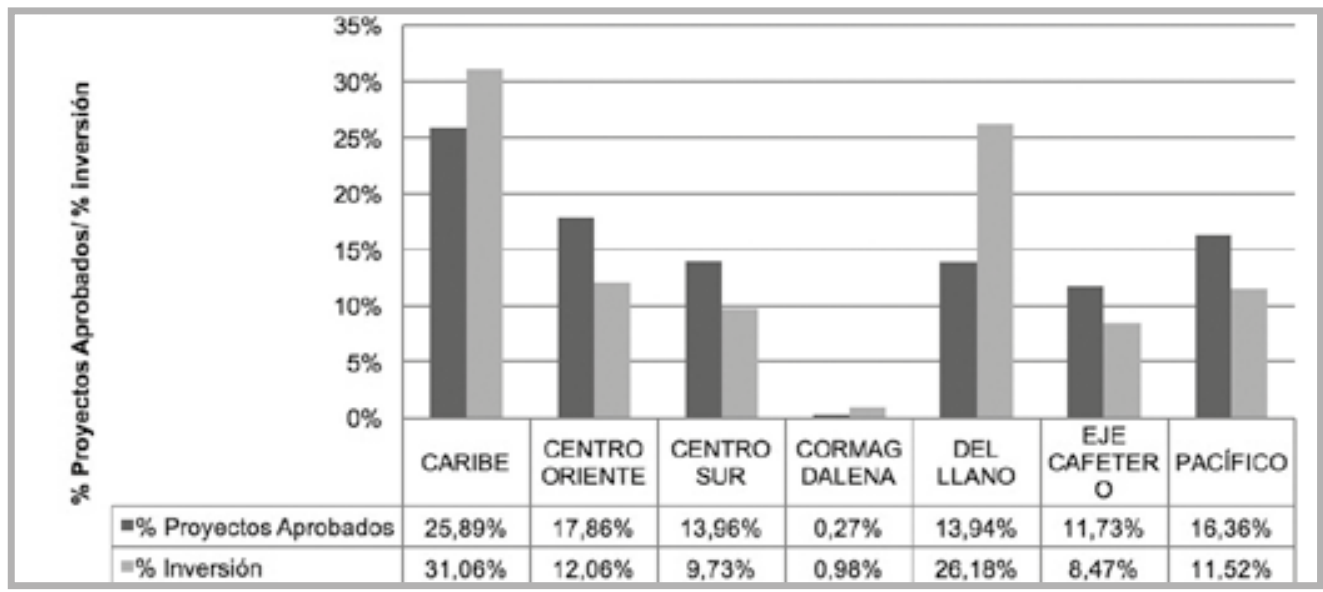

Figura 1.

Proporción de proyectos aprobados por cada región y porcentaje de Inversión.

Fuente: Autores. Información proveniente del Sistema de Monitoreo, Seguimiento, Evaluación y Control del SGR.

información publicada por el Ministerio de Vivienda en el diario El Espectador (2016), tan solo 2 de cada 10 personas en el área rural tienen acceso al agua potable, y en la cabecera municipal tan solo 7 de cada 10 accede a este servicio. Actualmente, Colombia ha declarado la emergencia social y sanitaria en el departamento de La Guajira. Según el Plan de Desarrollo de La Guajira, el departamento no solo se encuentra en el podio de la pobreza, sino que también es el líder en cifras de informalidad laboral (Riohacha, $81 \%$ ), lo que también lo hace campeón en inequidad, limitando el desarrollo económico y el crecimiento sostenible.

No obstante lo anterior, en el departamento de La Guajira se encuentra que el mayor monto de inversión -entre el año 2012 y el año 2015- se destina al sector educación, con $22,59 \%$ y qué el sector de agua potable y saneamiento básico se ubica en el quinto lugar, con $6,88 \%$.

Los anteriores ejemplos ponen en evidencia que las políticas no siempre son dirigidas a la necesidad de cada población objetivo, además de mostrar que no existe un patrón único en la asignación de los recursos del SGR así como en los procesos de toma de decisiones sobre las inversiones, por lo que su tratamiento debe darse usando técnicas de modelamiento y simulación para sistemas complejos.

\section{Justificación de la aplicación de dinámica de sistemas}

Simulación es la técnica numérica para conducir experimentos en una computadora digital. Estos experimentos comprenden ciertos tipos de relaciones matemáticas y lógicas, las cuales son necesarias para describir el comportamiento y la estructura de sistemas complejos del mundo real a través de largos periodos de tiempo. (Nailor, Balintfy, Burdick \& Chu 1971)

Sin duda alguna, los problemas sociales son sistemas complejos, ya que pueden ser descritos como un conjunto de elementos que se interrelacionan de modo no trivial en un todo orgánico y conforman un sistema organizado, como lo afirman Rodríguez y Leónidas (2011). La estructura compleja de los problemas sociales le otorga su particular comportamiento adaptativo, autoorganizado, emergente y no lineal, cualidades que son comunes en el problema a estudiaren este trabajo, que, por lo tanto, debe ser abordado con la metodología adecuada, ya que, como mencionan Izquierdo, Galán, Santos y Olmo (2008),“(...) la representación verbal de sistemas complejos carece de rigor para valorar su coherencia lógica y para generalizar, mientras que los modelos matemáticos suelen ser menos realistas debido a las fuertes restricciones que son impuestas...."

Easton (1992) asegura, en su obra, que la política es un proceso complejo que tiene unas entradas que generan diferentes salidas (o políticas autoritarias), por tanto, se trata de un sistema social abierto que se encuentra rodeado e influenciado por ambientes físicos, biológicos, sociales y psicológicos.

Reconociendo el ambiente político como un sistema social y con las implicaciones inherentes a su naturaleza, se decide abordar este problema a través de la dinámica de sistemas (DS) por las implicaciones no lineales y sociales expuestas anteriormente y sustentadas en los autores citados durante el desarrollo del texto. 


\section{Método}

Siguiendo la metodología del grupo de investigación SES (Mendez et al., 2015) se realizó una revisión bibliográfica de 50 autores, con el objetivo de conocer cuáles son las principales variables que afectan la formulación de la PP. De los autores consultados, 18 son de origen nacional, los 32 restantes de procedencia internacional, y de estos 22 son de Latinoamérica.

Con base en esta consulta se identificaron más de cincuenta parámetros entre variables y características que fueron clasificadas en un diagrama de causa-efecto, y organizadas en varios grupos según la relevancia y frecuencia de mención por parte de los expertos consultados.

Los grupos de variables son los siguientes:

Definición del problema: se relaciona con el problema específico que se quiere tratar, este agrupa el territorio, la población objetivo, el problema que afecta a la comunidad, entre otros.

Recursos: tanto económicos, como naturales y no tangibles (conocimiento, información).

Actores: son los interesados y participantes de la PP en general. Principalmente son la ciudadanía, el Estado, organismos internacionales, sector privado, los expertos, entidades gubernamentales, entre otros.

Aspecto técnico: herramientas, modelos y metodologías para formular la PP, como son la formulación, implementación y evaluación de la PP, el seguimiento y monitoreo de la PP, la normatividad, los sistemas de información y los Derechos Humanos.
Intereses: características e influencias que pueden tener los diferentes actores al momento de relacionarse para llegar a acuerdos, así como para tomar decisiones. Las variables principales son la igualdad, las metas y objetivos, el establecimiento de prioridades, la moral, la corrupción, entre otros.

Aspecto político: son todas las variables relacionadas con el ámbito político y que influyen en la elaboración de la PP. Estos son: gobernabilidad, democracia, el sistema político, la participación electoral, los partidos políticos y conexiones con programas del estado.

Con esta clasificación se procedió a realizar el pareto de frecuencia de cada grupo de variables, el cual se puede ver en la Figura 2. Allí se aprecia mejor la contribución de cada grupo de variables nombradas.

\section{Diagrama causal}

Existe un ciclo principal o bucle, que se puede definir como el proceso de la PP, que comienza con la identificación del problema, seguido de la formulación de la PP, y continúa con su implementación -a la cual se le realiza el seguimiento y monitoreo correspondiente-; de este proceso se obtienen resultados que ayudan a mantener en "el camino" la implementación. En la fecha de caducidad establecida para la PP se finaliza con la evaluación de los logros alcanzados, siendo este resultado un insumo para una nueva formulación de política en el mismo sector o con una problemática similar. Lo anterior se puede evidenciar en la Figura 3.

En la literatura poco se habla del bucle como tal; en la mayoría se estudia el ciclo como una serie de etapas lineales, que se suceden una tras otra, sin retorno. En este sentido, se pueden destacar dos de los documentos consultados para describir de forma más puntual este

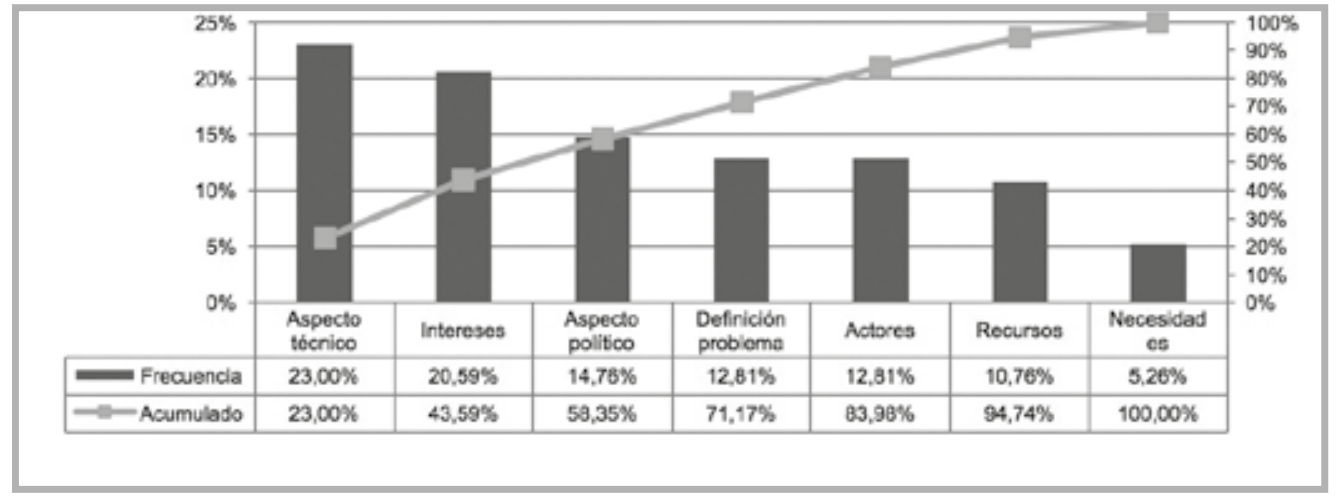

Figura 2.

Diagrama de Pareto agrupado.

Fuente: Autores 
bucle, el de la alcaldía Mayor de Bogotá -en el que se describe el ciclo de las PP a partir de cinco fases: identificación del problema, formulación de soluciones, toma de decisión, implementación y evaluación; la última fase se lleva a cabo "con el propósito de dar pie al reinicio de todo el ciclo con el fin de reajustar la respuesta (feedback) o suprimir la política (programtermination)" (Alcaldía Mayor de Bogotá. Secretaría de desarrollo Económico, 2009)-, y el escrito por Cardozo (2013), quien asegura que: El ciclo teórico de las políticas y programas públicos identifica el desarrollo de tres etapas fundamentales -formulación, implementación y evaluación- después de las cuales se reinicia un nuevo proceso, donde la formulación es retroalimentada con los resultados de la evaluación (reformulación)

La formulación de la política se inicia con la identificación y definición del problema que nace de necesidades y problemas previamente identificados por actores sociales. Luego, se evalúa si es pertinente la intervención de las autoridades del Estado; dicho de otro modo, si será inscrito en la agenda pública (Alcaldía Mayor de Bogotá. Secretaría de Desarrollo Económico, 2009). Vargas (2007) señala la importancia de esta etapa, ya que la definición del problema marca el posterior desarrollo de la política al reducir las posibles alternativas a considerar. Por lo tanto, se debe realizar una adecuada identificación de los actores relacionados con el problema que den sus opiniones y así construir una definición lo más cercana a la realidad.

Los actores tipificados por los diferentes autores pueden ser expertos provenientes de universidades y centros de investigación; la ciudadanía como población afectada; representantes de los partidos políticos, el Estado, representado por sus diferentes instituciones; representantes del sector privado; el sector internacional representado por organismos internacionales y las ONG y medios de comunicación.

Aldana (2012) asegura que previamente a la ejecución de la política, existe el proceso de asignación de los recursos de acuerdo a las prioridades establecidas para los problemas identificados por el Estado. Por su parte, Rodolfo Canto (2015) propone rediseñar la formulación de la PP al aprovechar la energía e influencia de la gente del común en la solución de los problemas que los aquejan, y el compromiso del Estado para tener en cuenta estos actores en la toma de decisiones inteligentes a través de deliberaciones razonables. Esta relación se puede apreciar en las conexiones entre los actores y la definición del problema, y en su formulación e implementación.

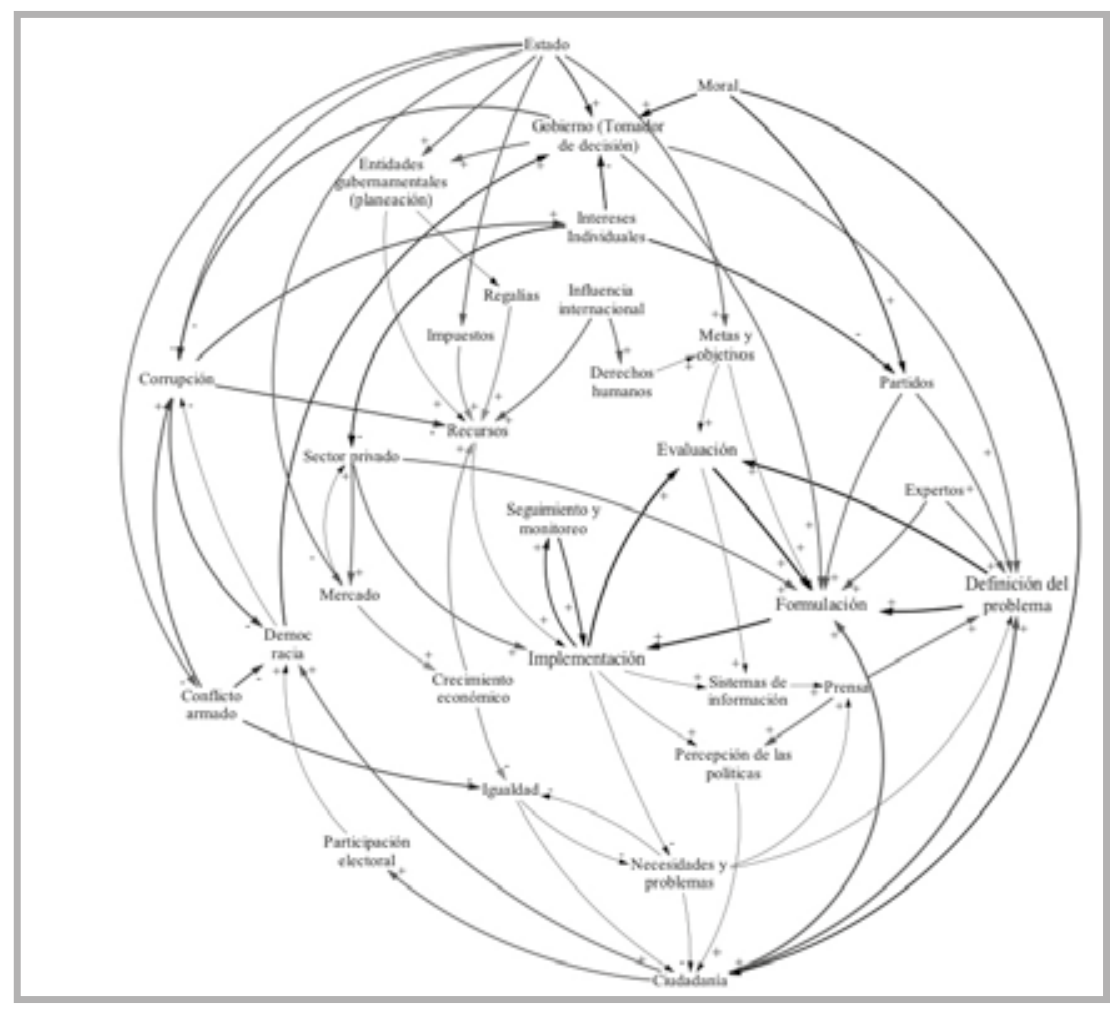

Figura 3.

Diagrama causal.

Fuente: Autores mediante Vensim ${ }^{\odot}$ 
Es primordial considerar a la población objetivo, sus costumbres y cultura, la ubicación geográfica y el contexto en el que esta se desarrolla en el problema identificado. Todo esto incide en la formulación de la PP, como lo asegura la Cepal (1997) en uno de sus reportes: "La formulación de PP que tomen en cuenta la evolución de la estructura demográfica por edades, su distribución espacial y las consecuencias sectoriales de las transformaciones demográficas a medio y a largo plazo, y que reconozca sus especificidades territoriales"..

En lo que respecta a la ciudadanía, es necesario incentivar la participación de esta, ya que muchas veces, debido a promesas incumplidas, como lo menciona Ortegón (2008), la mayoría de la población muestra un cierto grado de apatía y desinterés hacia las PP. Para evitar esto, se deben cumplir las expectativas de la población, con este fin se puede utilizar medios como la prensa. Vargas (2007) indica por qué se debe informar a la ciudadanía de forma clara, estableciendo canales de comunicación y relaciones adecuadas con la prensa y periodistas. Otro factor que afecta la ciudadanía es la percepción de igualdad, ya que unos altos niveles de pobreza y desigualdad, acompañado de un abandono del Estado, ocasionan una precaria democracia y baja participación ciudadana. Programa de las Naciones Unidas para el Desarrollo citado por Ortegón (2008).

Una variable importante (sobre todo en el caso colombiano) es el conflicto armado. Este contribuye a elevar la desigualdad generando pobreza, especialmente en zonas rurales, y afecta negativamente a la democracia además de incrementar la corrupción. Según López y Duncan, citado por Cendales, Mora \& Arroyo (2015), "La alianza entre los ejércitos rebeldes, narcotraficantes y políticos se ha hecho tan robusta en esta región que los grupos de poder, al cooptar los ejecutivos locales con el concurso del narcotráfico". Este mismo autor señala que los alcaldes buscan depredar los recursos luego de ser elegidos, afectando la democracia y aumentando las necesidades de los más vulnerables.

Respecto a los intereses individuales Roth (citado por la Alcaldía Mayor de Bogotá, Secretaría de desarrollo Económico, 2009) señala que "la representación de un problema es una construcción que resulta del conjunto de las luchas que los actores sociales y políticos libran para imponer una lectura de un problema que sea la más ventajosa posible para sus intereses". Estos intereses pueden ser afectados por la corrupción, de tal forma que se busque el beneficio individual y no el bienestar colectivo. Para Canto (2015) los racionamientos de orden moral son necesarios para la formulación de la PP, ya que está por encima de las ambiciones personales y busca el bienestar común.

\section{Resultados}

\section{Modelo de simulación}

A partir del diagrama causal se construye el modelo dinámico, que se divide en cuatro módulos distribuidos de acuerdo a la dinámica e interacción que se presenta entre sus variables, como se puede ver en las Figuras 4 y 5. La simulación será corrida desde el año 2012, por marcar el inicio del nuevo SGR y terminará en el año 2015, para contar con la posibilidad de comparar los resultados obtenidos en el modelo dinámico con lo sucedido en estos cuatro años.

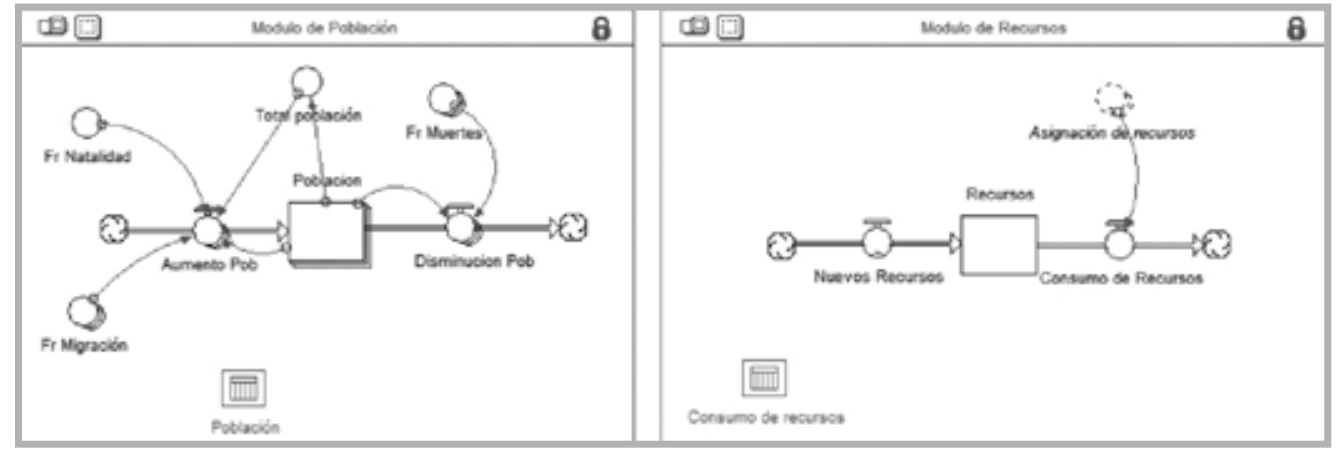

Figura 4.

Módulos de población y recursos en IThink ${ }^{\circledast}$

Fuente: Autores 


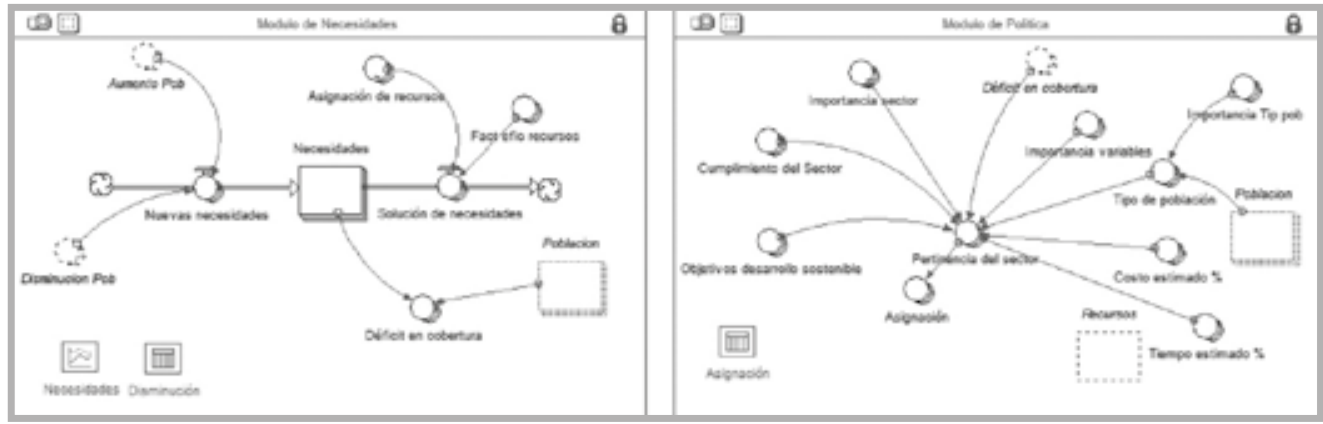

Figura 5.

Módulos de necesidades y PP en IThink

Fuente: Autores

Para probar la validez del modelo se trabaja el caso del departamento de La Guajira, ya que es una de las poblaciones con más necesidades básicas en el país.

\section{Módulo población}

Este módulo indica el comportamiento demográfico del departamento, el cual se distribuye en grupos de edades, como se encuentra en las series de población del DANE (Departamento Administrativo Nacional de Estadística, n. d.). A partir de esta distribución se conformaron cinco grupos de edades: de 0-4 años, denominada primera infancia; de 5-14, en edad escolar; 15-19 años, en edad escolar y laboral; 20-64 años, en edad laboral, y mayores a 65 , en edad avanzada.

La población se inicia con 846641 habitantes proyectados por el DANE para La Guajira en el 2011. Esta población crece con una tasa anual aproximada de 0.029para el quinquenio 2010-2015, y decrece para el rango de edad de cinco años. La cantidad de habitantes es afectada por una tasa de migración para el quinquenio y por las defunciones. (Departamento Administrativo Nacional de Estadística, 2007)

\section{Módulo de recursos económicos}

Este módulo trabaja con una tasa de entrada de dinero equivalente al aumento real de los cuatro años de estudio; ver Tabla 1. Por otro lado, se tiene una tasa de consumo de recursos de acuerdo a lo asignado a cada sector (variable de asignación). Esta variable es la conexión con los demás módulos y es clave en el modelo propuesto.

\section{Módulo de necesidades}

Se establecen seis necesidades ligadas a los sectores anteriormente expuestos; la justificación de su elección se basa en el cálculo de IPM (Índice de Pobreza
Tabla 1

Recursos económicos del SGR para La Guajira para cada año.

\begin{tabular}{cc}
\hline Año & Nuevos recursos \\
\hline $\mathbf{2 0 1 1}$ & 247691097130 \\
$\mathbf{2 0 1 2}$ & 233376189990 \\
$\mathbf{2 0 1 3}$ & 106463894553 \\
$\mathbf{2 0 1 4}$ & 179733600225 \\
$\mathbf{2 0 1 5}$ & 141413378106 \\
\hline
\end{tabular}

Fuente: Los Autores

Tabla 2

Déficit inicial y porcentaje de priorización definido para cada Necesidad

\begin{tabular}{lcc}
\hline Necesidad & Valor inicial* & Importancia \\
\hline Educación & $23,0 \%^{18}$ & $5,21 \%$ \\
Trabajo & $12,9 \%^{2}$ & $8,80 \%$ \\
Salud & $11,8 \%^{3}$ & $23,28 \%$ \\
Acueducto y alcantarillado & $67,8 \%^{4}$ & $44,33 \%$ \\
Vivienda & $17,01 \%^{5}$ & $15,33 \%$ \\
Transporte & $40,9 \%^{* *}$ & $3,04 \%$ \\
\hline
\end{tabular}

Nota: *Déficit en la cobertura de la necesidad al término del año 20101. (Secretaría de Educación del Departamento de La Guajira, 2012)2. (Departamento Administrativo Nacional de estadística \& Banco de la República, 2012)3. (Ministerio de Salud y Protección Social, 2011)4. (Piamba Escobar, 2014)5. (Ministerio de Vivienda, 2014). **Se establece por la relación entre los kilómetros de vías pavimentadas por cada millón de habitantes (Departamento de La Guajira, 2009) y el estándar mundial (Garay, n.d.).

\section{Fuente: Los Autores}

Multidimensional) para Colombia, que está conformado por cinco dimensiones: 1. condiciones educativas del hogar; 2 . condiciones de la niñez y juventud; 3 . trabajo; 
4. salud; 5 . servicios públicos domiciliarios y condiciones de la vivienda (Departamento Nacional de Planeación, Dirección de Desarrollo Social y Subdirección de Promoción Social y Calidad de Vida, 2014). Las dos primeras se traducen en la necesidad de educación, las dos siguientes conservan su nombre por coincidir con su denominación, y la quinta es equivalente a las necesidades básicas de vivienda y acueducto y alcantarillado. La sexta y última necesidad es transporte, elegida a criterio de los autores como mecanismo de contraste con la inversión real.

Las necesidades están representadas en un arreglo de niveles cuyas tasas de crecimiento se ven afectadas por el aumento en la población. Cada necesidad crece de acuerdo a la cantidad de habitantes que la demandan, por lo cual cada sector será afectado según los rangos de edad establecidos. El objetivo es llegar a un déficit de cobertura igual a 0 , para lo cual se mide el déficit anual en el cubrimiento de cada necesidad. La tasa de salida (solución de necesidades) va en aumento según la asignación de recursos que se establezcan en el módulo de la PP.

Teniendo en mente el criterio de supervivencia, se procede a priorizar las necesidades a través de la técnica de proceso analítico jerárquico (Analytic Hierarchy Process, AHP) en la que, básicamente, se asigna una "calificación" numérica a la influencia entre alternativas respecto a un criterio o atributo (Moreno Jiménez, n. d.). En la Tabla 2 se muestran las asignaciones correspondientes a cada necesidad.

\section{Módulo de política pública}

El último módulo está conformado por siete criterios de decisión elegidos a partir de la revisión de autores, previamente a la construcción del diagrama de Pareto presentado en el capítulo anterior. Estos criterios también fueron priorizados por el método AHP, evaluando la influencia que tienen frente a la satisfacción de las necesidades. Los criterios y la importancia correspondiente se muestran en la Tabla 3.

El déficit de cobertura es una variable cuyo valor es extraído del módulo de Necesidades. El criterio Cumplimiento del sector mide la frecuencia con que se cumplieron los tiempos establecidos para el desarrollo de los proyectos(Departamento Nacional de Planeación, 2015a).

Los Objetivos de Desarrollo Sostenible (ODS) para Colombia se derivan de los ODS propuestos por la ONU, son diecisiete propósitos dirigidos a reducir la pobreza, proteger el planeta y asegurar la prosperidad para todos (Programa de las Naciones Unidas para el Desarrollo, 2015). Cada objetivo se evalúa frente a las seis necesidades para establecer la pertinencia individual y su participación integral para cada necesidad. Los resultados se muestran en la Tabla 4.

Tabla 3

Criterios de decisión del módulo PP y porcentajes de priorización correspondiente

\begin{tabular}{ll}
\hline Criterios de decisión & Importancia \\
\hline Déficit cobertura & $28,7 \%$ \\
Cumplimiento sector & $2,4 \%$ \\
Objetivos DS & $16,5 \%$ \\
Tipo de población & $38,7 \%$ \\
Costo estimado & $6,3 \%$ \\
Tiempo estimado & $7,4 \%$ \\
\hline
\end{tabular}

Fuente: Los Autores

Tabla 4

Importancia ponderada de las Necesidades frente a los ODS

\begin{tabular}{ll}
\hline Necesidad & Importancia integral \\
\hline Salud & $64,41 \%$ \\
Educación & $80,59 \%$ \\
Vivienda & $58,82 \%$ \\
Acueducto y Alcantarillado & $78,24 \%$ \\
Trabajo & $56,47 \%$ \\
Transporte & $44,71 \%$ \\
\hline
\end{tabular}

Fuente: Los Autores

El criterio del tipo de población se refiere a la importancia que tiene cada porción de la población de acuerdo al rango de edad al que pertenece, principalmente evaluando el nivel de dependencia que supone cada rango de edad. Teniendo en mente este criterio de dependencia, se usa el método de AHP para conseguir los resultados de prioridad expuestos en la Tabla 5.

Tabla 5

Porcentaje de importancia por rango de edades establecidas

\begin{tabular}{ll}
\hline Rango de edades & Resultado del AHP \\
\hline $\mathbf{0}$ a $\mathbf{5}$ & $40,71 \%$ \\
$\mathbf{5}$ a $\mathbf{1 4}$ & $14,48 \%$ \\
$\mathbf{1 5}$ a $\mathbf{1 9}$ & $7,84 \%$ \\
$\mathbf{2 0}$ a 64 & $2,94 \%$ \\
Mayor a 65 & $34,02 \%$ \\
\hline
\end{tabular}

Fuente: Los Autores 
Tanto el costo estimado como el tiempo estimado son calculados por datos históricos de los proyectos aprobados entre el 2012-2015. (Departamento Nacional de Planeación, 2016)

\section{Tabla 6}

Tiempo estimado, Costo estimado y Cumplimiento del sector para cada necesidad

\begin{tabular}{lccc}
\hline Necesidad & $\begin{array}{c}\text { Tiempo } \\
\text { estimado } \\
\text { (meses) }\end{array}$ & $\begin{array}{c}\text { Costo estimado } \\
\text { (dinero en pesos } \\
\text { colombianos } \\
\text { persona) }\end{array}$ & $\begin{array}{c}\text { Cumplimiento } \\
\text { del sector }\end{array}$ \\
\hline Educación & 35,31 & 1908872,36 & $74,27 \%$ \\
Vivienda & 40,09 & 10965865,67 & $72,68 \%$ \\
Salud & 65,77 & 4077989,21 & $73,25 \%$ \\
Acueducto y & 78,46 & 1286498,35 & $74,55 \%$ \\
alcantarillado & & & \\
Trabajo & 60,73 & 2181450,79 & $71,96 \%$ \\
Transporte & 74,17 & 1195020,42 & $73,49 \%$ \\
\hline
\end{tabular}

\section{Fuente: Los Autores}

Estos porcentajes de importancia se multiplican por la población por tipo con el objetivo de que sea variable en cada año.

Todo este proceso se hace con el fin de calcular el porcentaje de los recursos que deberían ser asignados a cada necesidad, para lo cual se calculó de la siguiente forma:
Dónde:

$I_{\mathrm{i}}$ : Importancia para todos los criterios $\mathrm{i}$, siendo $\mathrm{i}=\mathrm{D}, \mathrm{CS}, \mathrm{O}, \mathrm{Tp}, \mathrm{C}$ y $\mathrm{T}$

$D_{n, t}:$ Déficit para cada necesidad $n$ en el tiempo $t$

$\mathrm{CS}_{\mathrm{n}}$ : Cumplimiento del sector para cada necesidad $\mathrm{n}$

$\mathrm{O}_{\mathrm{n}}:$ Importancia de los ODS para cada necesidad $\mathrm{n}$

$\mathrm{Tp}_{\mathrm{r}, \mathrm{t}}:$ Importancia de cada rango de edadesr en el tiempo $\mathrm{t}$

$\mathrm{ri}_{\mathrm{n}^{\prime}}, \mathrm{rs}_{\mathrm{n}}$ : Rango de edad inferior y superior para cada necesidad $\mathrm{n}$

$C_{n}$ : Costo estimado por persona para suplir la necesidad $n$

$\mathrm{T}_{\mathrm{n}}$ :Tiempo estimado para suplir la necesidad $\mathrm{n}$

$\mathbb{I N}_{\mathrm{n}}$ : Importancia de cada necesidad $\mathrm{n}$

\section{Análisis}

Los resultados de la variable asignación en el software IThink se muestran en la Figura 6, y la variable del comportamiento del déficit, en la Figura 7.

$$
\left(I C_{D} * D_{n, t}+I C_{C S} * C S_{n}+I C_{o} * O_{n}+I C_{T p} \cdot \sum_{r i_{n}}^{r s_{n}} T p_{r, t}-I C_{C} * C_{n}-I C_{\tau} * T_{n}\right) * I N_{n} \forall n, t
$$

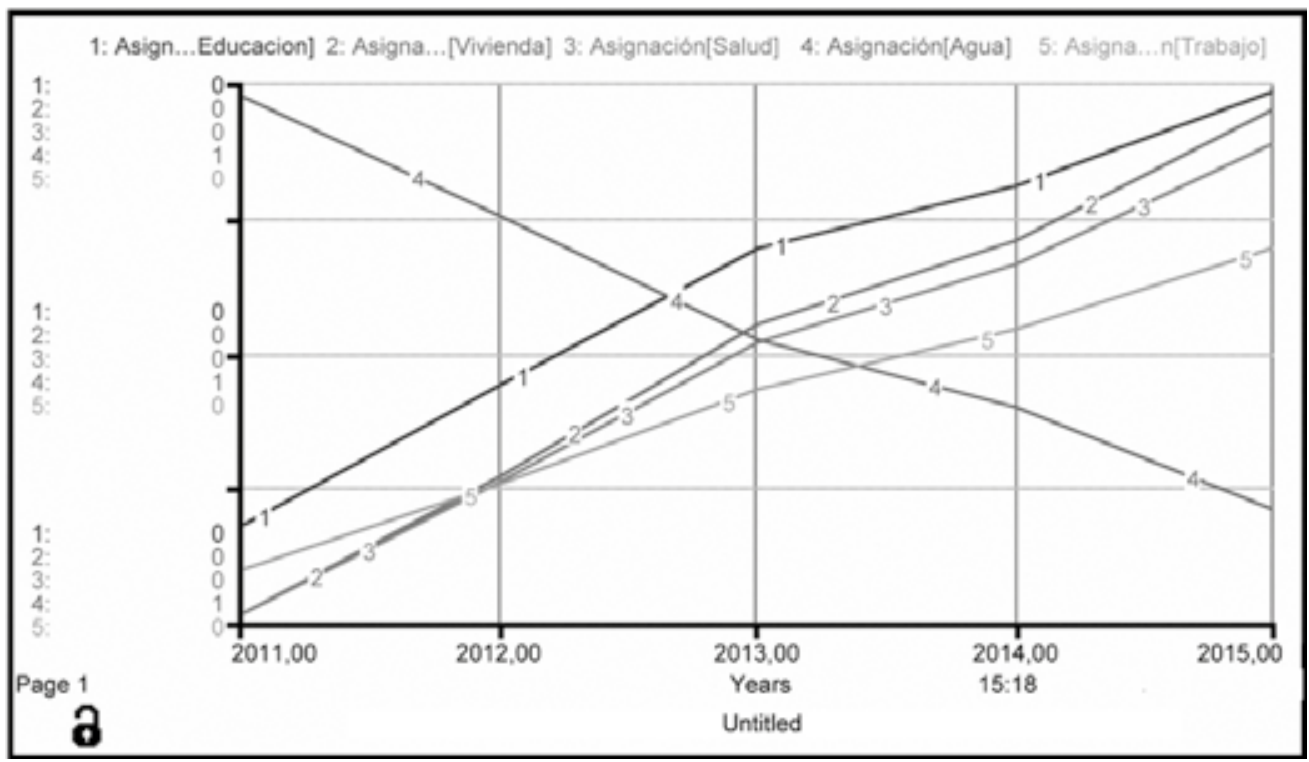

Figura 6.

Porcentaje de asignación de recursos para cada necesidad cada año. Fuente: Autores. Mediante IThink ${ }^{\circledast}$. 
En la Figura 6 se puede ver que el mayor porcentaje de recursos se destina a suplir la necesidad de acueducto y alcantarillado, lo que provoca una disminución considerable del déficit frente a las demás necesidades, las cuales registran un pequeño aumento a lo largo de los años. A medida que la necesidad de acueducto es satisfecha, se producen aumentos en la asignación de recursos a las otras necesidades, como lo evidencian las Figuras 6 y 7 del modelo en IThink.

A continuación, se presenta la asignación de los recursos por el SGR a cada sector en el departamento de La Guajira (Departamento Nacional de Planeación, 2015b):
Tabla 7

Proporción de asignación de recursos para cada necesidad cada año

\begin{tabular}{lccccc}
\hline Años & Inicial & $\mathbf{2 0 1 2}$ & $\mathbf{2 0 1 3}$ & $\mathbf{2 0 1 4}$ & $\mathbf{2 0 1 5}$ \\
\hline Asignación (Educación) & 0,04 & 0,05 & 0,05 & 0,06 & 0,06 \\
Asignación (Vivienda) & 0,09 & 0,10 & 0,11 & 0,11 & 0,12 \\
Asignación (Salud) & 0,13 & 0,14 & 0,15 & 0,17 & 0,18 \\
Asignación (Agua) & 0,68 & 0,64 & 0,61 & 0,58 & 0,56 \\
Asignación (Trabajo) & 0,04 & 0,04 & 0,04 & 0,05 & 0,05 \\
Asignación (Transporte) & 0,03 & 0,03 & 0,03 & 0,03 & 0,04 \\
\hline
\end{tabular}

Fuente: Los Autores

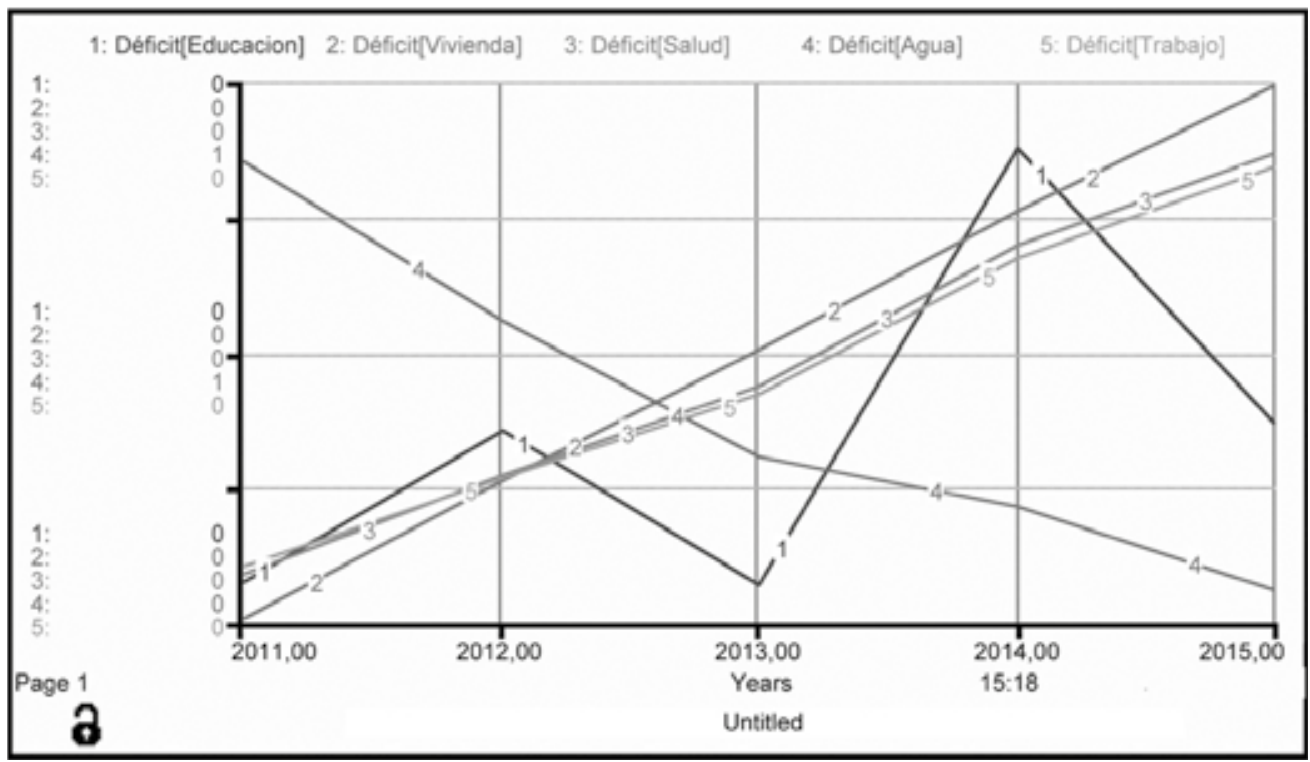

Figura 7.

Déficit para cada una de las necesidades anual.

Fuente: Autores. Mediante IThink ${ }^{\circledast}$.

Tabla 8

\section{Déficit para cada necesidad cada año}

\begin{tabular}{lccccc}
\hline Años & Inicial & $\mathbf{2 0 1 2}$ & $\mathbf{2 0 1 3}$ & $\mathbf{2 0 1 4}$ & $\mathbf{2 0 1 5}$ \\
\hline Déficit (Educación) & 0,23 & 0,23 & 0,23 & 0,24 & 0,23 \\
Déficit (Vivienda) & 0,17 & 0,18 & 0,19 & 0,20 & 0,21 \\
Déficit (Salud) & 0,12 & 0,12 & 0,13 & 0,13 & 0,14 \\
Déficit (Agua) & 0,68 & 0,53 & 0,41 & 0,36 & 0,28 \\
Déficit (Trabajo) & 0,13 & 0,13 & 0,13 & 0,14 & 0,14 \\
Déficit (Transporte) & 0,41 & 0,41 & 0,41 & 0,42 & 0,42 \\
\hline
\end{tabular}

Fuente: Los Autores
Tabla 9

Porcentaje de asignación real de los recursos para cada necesidad

\begin{tabular}{lcccc}
\hline \multicolumn{5}{c}{ Asignación } \\
\hline Necesidad & $\mathbf{2 0 1 2}$ & $\mathbf{2 0 1 3}$ & $\mathbf{2 0 1 4}$ & $\mathbf{2 0 1 5}$ \\
Educación & $15,17 \%$ & $60,58 \%$ & $50,64 \%$ & $25,34 \%$ \\
Vivienda & $0,67 \%$ & $2,76 \%$ & $9,83 \%$ & $14,42 \%$ \\
Salud & $20,46 \%$ & $6,65 \%$ & $0,84 \%$ & $15,01 \%$ \\
Acueducto y & $12,13 \%$ & $6,08 \%$ & $16,88 \%$ & $26,02 \%$ \\
alcantarillado & & & & \\
Trabajo & $0,00 \%$ & $4,18 \%$ & $0,00 \%$ & $0,29 \%$ \\
Transporte & $51,57 \%$ & $19,75 \%$ & $21,81 \%$ & $18,91 \%$ \\
\hline
\end{tabular}

Fuente: Los Autores 
Los datos fueron agregados al modelo con el fin de evaluar y comparar la eficiencia de las asignaciones en la reducción del déficit.

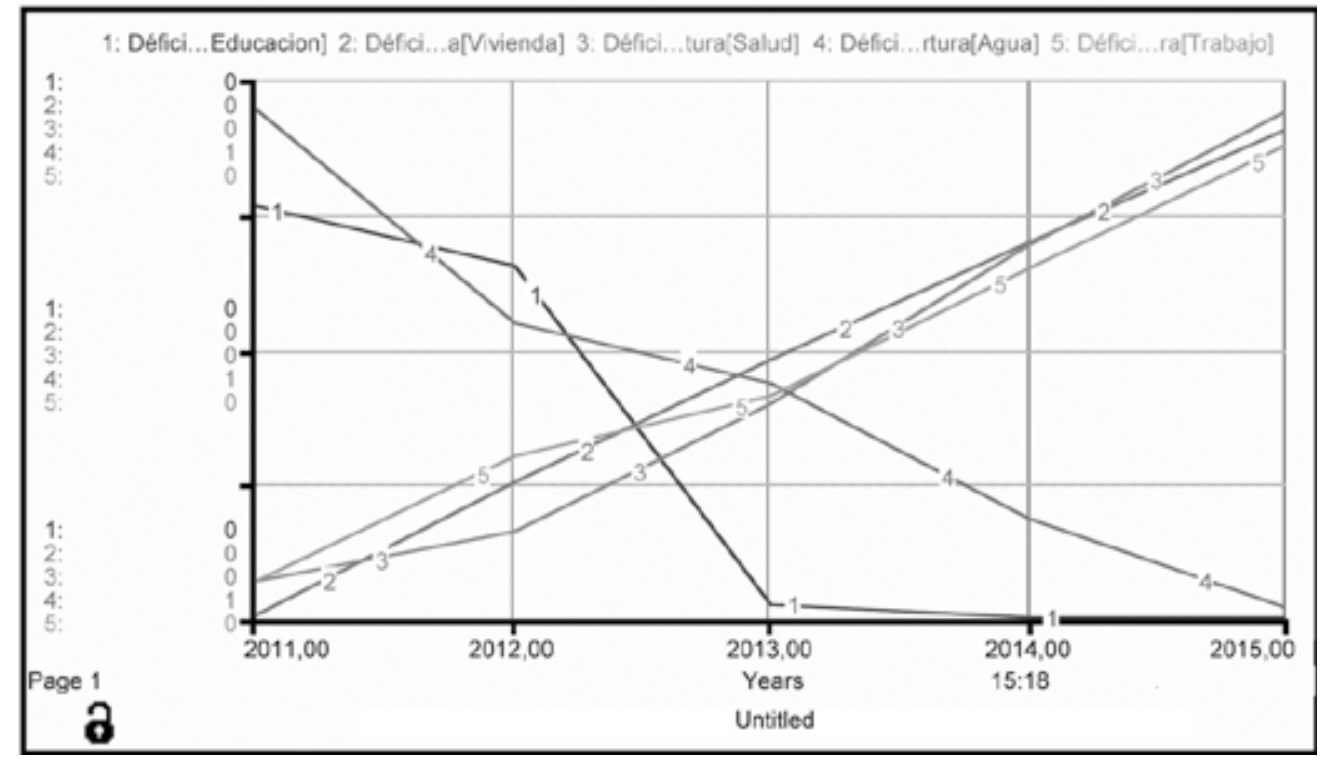

Figura 8.

Déficit para cada una de las necesidades con la asignación real

Tabla 10

\section{Déficit para cada necesidad con la asignación real}

\begin{tabular}{lccccc}
\hline Años & Inicial & $\mathbf{2 0 1 2}$ & $\mathbf{2 0 1 3}$ & $\mathbf{2 0 1 4}$ & $\mathbf{2 0 1 5}$ \\
\hline Déficit (Educación) & 0,23 & 0,20 & 0,01 & 0,00 & 0,00 \\
Déficit (Vivienda) & 0,17 & 0,18 & 0,20 & 0,21 & 0,22 \\
Déficit (Salud) & 0,12 & 0,12 & 0,13 & 0,15 & 0,16 \\
Déficit (Agua) & 0,68 & 0,66 & 0,66 & 0,65 & 0,64 \\
Déficit (Trabajo) & 0,13 & 0,14 & 0,15 & 0,16 & 0,18 \\
Déficit (Transporte) & 0,41 & 0,32 & 0,30 & 0,29 & 0,29 \\
\hline
\end{tabular}

\section{Fuente: Los Autores}

Al comparar la Figura 6 y la Tabla 7 se puede ver que existe una gran diferencia en los porcentajes de asignación de cada sector, especialmente en el acueducto y alcantarillado, y en transporte, ya que en el modelo se dio una mayor prioridad a suplir la necesidad de agua potable generando mayores recursos para este sector. En la asignación real del sistema se designó una gran cantidad de recursos a la necesidad de transporte, que aunque es importante, no es fundamental para la vida, lo que genera que al introducir los datos reales al modelo resulte una reducción muy pequeña para el déficit del sector acueducto y alcantarillado, como se puede ver en la Figura 8.

Además, existe otra diferencia en las asignaciones, presente en el sector de educación, en el cual se le asigna hasta un $60,58 \%$ en el 2013 , frente al 3,6\% asignado por el modelo. Esto se debe a que no se le dio mayor prioridad a la educación ya que no es una necesidad indispensable en el corto plazo.

Finalmente, se observa que los déficits, a excepción del sector de acueducto, no disminuyen con el paso de los años, ya que los recursos no son suficientes para cubrir adecuadamente varias necesidades a la vez.

\section{Discusión}

En Colombia se evidencian diariamente problemas relacionados con el conflicto interno armado, la sequía o las fuertes lluvias con grandes pérdidas en la economía del país; niños y niñas en desnutrición y pésimas condiciones de saneamiento básico, entre otros. Existen otros elementos estructurales que no permiten el completo desarrollo del territorio, relacionados con la equivocada administración de los recursos, como los obtenidos de las regalías.

Las decisiones de inversión en el SGR no se están priorizando de manera correcta, ya que no consideran las condiciones de complejidad delos territorios, la cantidad de actores, intereses y variables que se relacionan, equivocando la distribución y control de estos recursos. 
El SGR implementado a partir del 2012 ha mostrado importantes logros en las inversiones de proyectos de inversión que están correctamente estructurados; no obstante, la mayor debilidad del SGR consiste en que no existe un modelo para la toma de decisiones que incorpore elementos de complejidad e incertidumbre. Con la propuesta de este modelo que integra AHP y simulación permite decidir cuál proyecto es el que se debe estructurar y ejecutar a fin de generar un mayor impacto en la población objetivo, disminuir las brechas sociales y mejorar las condiciones de vida de las comunidades.

Se evidencia la importancia del modelo como herramienta para comprender y establecer las relaciones no lineales de un sistema complejo, cambiante en el tiempo y que facilita conocer los posibles resultados al efectuar diferentes alternativas de inversión en los sectores propuestos ayudando al proceso de toma de decisiones.

A futuro, se desea desarrollar un modelo más complejo, que no solo escoja los sectores sino los proyectos a apoyar y así se tomen decisiones mejor ajustadas a la realidad, además de poder incluir todos los sectores que abarca el SGR.

\section{Referencias}

Alcaldía Mayor de Bogotá. Secretaría de desarrollo Económico. (2009). Marco conceptual y metodológico para la formulación de políticas publicas en el sector desarrollo económico. Recuperado de: https://www. google.com.co/QFggIMAA\&url=http\%3A\%2F\%2Fwww. d e s a r r o l l o e co nom i co.gov. co $\% 2 \mathrm{Fsitiodesarrolloold} \% 2 \mathrm{Findex}$. hp\%2Fdocumentos\%2Fcategory\%2F93-produccionintele ctual\%3Fdownload\%3D1865\%3Adocformulacionpolitica spublicas\&usg=AFQjCNECxX7fUGwgZH25qITMGJnjR3mZ fg\&sig2=e6qmUfOnYaDy00L_4j_HpA .

Aldana, D. (2012). Documentación de modelos pertinentes para el diseño del Sistema General de Regalías. Ministerio de Hacienda y Crédito Público.

Canto, R. (2015). Políticas públicas, racionalidad y razón. Tópicos, (49), 259-290. doi: http://dx.doi.org/10.21555/ top.v0i49.752

Cardozo, M. (2010). Gestión pública: complejidad y simulación. Administración y Organizaciones, 37-52.

Cardozo, M. (2013). De la evaluación a la reformulación de políticas públicas. Política y cultura, (40), 123-149.
Cendales, A., Mora, J. \& Arroyo, S. (2015). Sobre las democracias locales en el Pacífico colombiano y su incidencia en la política pública de agua potable durante el periodo 20082011. Lecturas de Economía, (83), 161-192. doi: http:// dx.doi.org/10.17533/udea.le.n83a06.

Comisión Económica para América Latina y el Caribe. (1997). Notas para el estudio del proceso de gestión pública en el campo de las políticas sociales. Recuperado de http:// repositorio.cepal.org/bitstream/handle/11362/30857/ S9708604_es.pdf?sequence=1\&isAllowed=y

Departamento Administrativo Nacional de Estadística. (s.f.). Estimaciones de la Migración. 1985-2005 y proyecciones 2005-2020, Nacionales y Departamentales. Recuperado de http://www.dane.gov.co/index.php/estadisticas-portema/demografia-y-poblacion/series-de-poblacion

Departamento Administrativo Nacional de Estadística. (s.f.). Estimaciones de Población 1985-2005 y proyecciones de población 2005-2020. Recuperado de http://www.dane. gov.co/index.php/estadisticas-por-tema/demografia-ypoblacion/series-de-poblacion

Departamento Administrativo Nacional de Estadística. (2007). Tablas abreviadas de mortalidad nacionales y departamentales 1985-2020. Recuperado de http:// www.dane.gov.co/files/investigaciones/poblacion/ royepobla06_20/8Tablasvida1985_2020.pdf

Departamento Administrativo Nacional de Estadística \& Banco de la República. (2012). Informa de Coyuntura Económica Regional. Recuperado de http://www.dane.gov.co/files/ icer/2011/laguajira_icer_11.pdf

Departamento de La Guajira. (2009). Plan vial departamental de La guajira. Recuperado de https://dirinfra.mintransporte. gov.co/PVR_DATA/DOCUMENTS/plan_guajira.pdf

Departamento Nacional de Planeación. (2012). Guía para la Evaluación de Políticas Públicas. Recuperado de https:// colaboracion.dnp.gov.co/CDT/Sinergia/Documentos/ Cartilla\%20Guia\%20para\%20Seguimiento\%20y\%20 Evaluaci\%C3\%B3n\%20Ago\%2013.pdf

Departamento Nacional de Planeación. (2015 a). Índice de gestión de proyectos de regalías.

Departamento Nacional de Planeación. (2015 b). Relación de proyectos aprobados a 15 oct 2015 Gesproy-SGR.

Departamento Nacional de Planeación. (2016 a). Mapa Regalías. Recuperado de http://maparegalias.sgr.gov.co/\#/ 
Departamento Nacional de Planeación. (2016b). Sistema de información y consulta Distribuciones Recursos Territoriales. Recuperado de https://sicodis.dnp.gov.co/ logon.aspx?ReturnUrl=\%2f

Departamento Nacional de Planeación, Dirección de Desarrollo Social \& Subdirección de Promoción Social y Calidad de Vida. (2014). Índice de Pobreza Multidimensional ( IPM-Colombia ) 1997-2008 y meta del PND. Recuperado de https://colaboracion.dnp.gov.co/CDT/Estudios\%20 Econmicos/\%C3\%8Dndice\%20de\%20Pobreza\%20 Multidimensional\%20(IPM-Colombia)\%201997-2008.pdf

Easton, D. (1992). Categorías para el análisis sistémico de la política. Diez Textos Básicos de Ciencia Política (p 221230). Recuperado de http://pendientedemigracion.ucm. es/info/cpuno/asoc/profesores/lecturas/easton.pdf

El Espectador. (15 de febrero de 2016). La Guajira tendrá 90\% de cobertura de agua potable en 2018: Minvivienda. Recuperado de http://www.elespectador.com/noticias/ nacional/guajira-tendra-90-de-cobertura-de-aguapotable-2018-min-articulo-616731

Garay, L. (1998). Colombia: estructura industrial e internacionalización 1967-1996. Recuperado de https:// colaboracion.dnp.gov.co/CDT/Desarrollo\%20Empresarial/ Colombia $\% 20$ Estructura $\% 20$ Industrial $\% 20$ e $\% 20$ Internacionalizaci\%C3\%B3n.pdf

Izquierdo, R., Galán, M., Santos, I. \& Olmo, R. (2008). Modelado de sistemas complejos mediante simulación basada en agentes y mediante dinámica de sistemas. Empiria, (16), 85-112. Recuperado de http://revistas.uned.es/index. php/empiria/article/viewFile/1391/1286

Méndez, G., Álvarez, P., \& López, S. (2015). Simulation of Social Problems using System Dynamics. In Engineering Applications-International Congress on Engineering (WEA).

MinisteriodeSaludyProtecciónSocial.(2011).CoberturaenSalud año 2011. Recuperado de https://www.minsalud.gov.co/ salud/Paginas/berturasdelR\%C3\%A9gimenSubsidiado. aspx

Ministerio de Vivienda. (2014). Déficit habitacional en Colombia cayó $46,3 \%$ gracias a los programas de vivienda que adelanta el gobierno nacional. Recuperado de http://www. minvivienda.gov.co/sala-de-prensa/noticias/2014/abril/ d\%C3\%A9ficit-habitacional-en-colombia-cay\%C3\%B3-463-gracias-a-los-programas-de-vivienda-que-adelanta-elgobierno-nacional
Moreno, J. (s.f.). El proceso analítico jerárquico ( ahp). Metodología y aplicaciones. Recuperado de http://www. uv.es/asepuma/recta/extraordinarios/Vol_01/02t.pdf

Nailor, T., Balintfy, J., Burdick, D., Chu, K (1971). Técnicas de simulación en computadoras, México DF, México: Limusa.

Organización de Naciones Unidas. (2010). Simulador de Políticas Públicas manual metodológico. Recuperado de http:// docplayer.es/1009993-Simulador-de-politicas-publicasmanual-metodologico-area-de-gestion-y-evaluacion-delestado-oficina-de-planeamiento-y-presupuesto.html

Ortegón, E. (2008). Guía sobre diseño y gestión de la política pública (Colciencias). Bogotá. Recuperado de http://ielat. com/inicio/repositorio/guia_gestion_politicas_publicas_ ortegon.pdf

Piamba, E. (2014). Disponibilidad, cantidad y calidad del recurso hídrico en La Guajira en el siglo XXI. (Tesis de especialización). Universidad Nacional de Colombia, Bogotá.

Programa de las Naciones Unidas para el Desarrollo. (2015). Objetivos de Desarrollo Sostenible, Colombia. Recuperado de http://www.co.undp.org/content/dam/colombia/ docs/ODM/undp-co-ODSColombiaVSWS-2016.pdf

Rodríguez, Z. \& Leónidas, A. (2011). Teorías de la complejidad y Ciencias Sociales: Nuevas estrategias epistemológicas y metodológicas. Nómadas, 30(2), 1-20. Recuperado de http://doi.org/10.5209/rev_NOMA.2011.v30.n2.36562

Secretaría de Educación del Departamento de La Guajira. (2012). Plan de cobertura educativa de los 12 municipios no certificados del departamento de La Guajira 2013 - 2014. Recuperado de http://sed-laguajira.gov.co/ apc-9383165383431373133626231356431/plan-decobertura.pdf

Segura, M. (2009). ¿Puede gestionarse la complejidad de los problemas sociales? Aportaciones de la teoría de la complejidad a la formulación de políticas públicas. Nómadas, 23(3), 273-289.

Vargas, A. (2007). Análisis de las políticas públicas. Perspectivas, (19), 127-136. Recuperado de http://www.redalyc.org/ pdf/4259/425942453011.pdf 